\title{
Effect of Residual of Self-Interference in Performance of Full-Duplex D2D Communication
}

\author{
Samad Ali, Amin Ghazanfari, Nandana Rajatheva, Matti Latva-aho \\ Centre for Wireless Communications, University of Oulu, P.O. Box 4500, FIN-90014, Oulu, Finland. \\ \{saali, aghazanf, rrajathe, matla\}@ee.oulu.fi
}

\begin{abstract}
This paper studies possibility of using full-duplex (FD) radios in underlay device-to-device communication (D2D) in cellular networks. We consider a cellular system with one D2D pair and one cellular user. Cellular user is sharing the radio resources with D2D link which is equipped with full-duplex radios. The problem of sum-power minimization of cellular system and D2D link both in uplink and downlink period is considered. Considering the interference caused because of exploiting the same radio resources, for uplink period, we use fixed point iterations to solve the optimization problem to calculate transmit powers of users. To design the optimal receiver in the base station, linear minimum mean squared error method is used. In the downlink, to calculate optimal transmit precoder at base station and optimal D2D transmit powers, the optimization problem is formulated as a second order cone problem (SOCP) and solved using CVX in Matlab. Since the available full-duplex radios are not able to cancel the self-interference completely, residual of self-interference is considered in D2D receivers. Performance of the full-duplex D2D with different amounts of self-interference cancelation is compared to that of half-duplex D2D. Results show that full-duplex radios with $110 \mathrm{~dB}$ self-interferece cancelation can provide double the throughput for D2D compared to halfduplex radios.
\end{abstract}

\section{INTRODUCTION}

Increasing demand for wireless communication requires new solutions and technologies to provide services for end users. Technologies like multiple-input multiple-output (MIMO), direct device-to-device communication (D2D), massive MIMO, in-band full-duplex radios (FD), etc. are some of the new solutions to address the problem of radio spectrum congestion. Among these, FD radios can give a high performance boost due to the fact that it requires half of the resources that conventional time-division-duplex (TDD) or frequencydivision-duplex (FDD) systems need. In FD radios, two way communication between nodes happens simultaneously on the same frequency band. The problem in FD radio design is that receiver of each node will receive the transmitted signal from its own transmitter. This self-interference (SI) is very large and this makes it impossible to recover the signal of interest. To design FD radios, this large self-interference needs to be canceled. Several FD radio systems with different amounts of self-interference cancelation have been reported in [1], [2], [3], [4], [5]. FD multi-antenna system for wi-fi systems has been designed and implemented in [6]. In [5] authors have presented design and implementation of single antenna fullduplex radios for WiFi. Using novel analog and digital selfinterference cancelation techniques, FD radio in that work can cancel up to $110 \mathrm{~dB}$ of self-interference and provide almost double the throughput compared to half-duplex radios. Based on the same FD radios, FD MIMO radio is also designed and implemented in [7], which almost is an ideal radio. These recent works shows that FD radio systems are feasible for the systems with small transmit power.

One of the possible application areas of these already designed radios is device-to-device communication (D2D) because of small transmit power of D2D systems. D2D communication is considered as a promising technology component in future cellular systems [8], [9]. In D2D, users in close proximity communicate directly without going through base station. D2D users can communicate using unlicensed bands (outband D2D) or licensed bands (inband D2D). In inband, D2D pair can use its own dedicated resource (overlay), or share the radio resources with one or more cellular users (underlay). While D2D is sharing the resources with other cellular users, both cellular users and D2D pair will face interference from this resource sharing. Feasibility of underlay D2D communication in LTE-A has been studied in [10] where authors show that because of flexibility of time and frequency resource allocation in LTE-A, it is possible to share the resources with D2D communication. Authors in [11] propose D2D mode selection considering the interferences between D2D and cellular users and quality of D2D and cellular users. A practical and optimal mode selection in presented and evaluated in [12]. In [13] authors have introduced interferencelimited-area method to guarantee the quality of service in D2D communication. In this method an area around D2D users will be found in which the interference on D2D users is higher than a predefined threshold and users outside this area are selected for resource sharing. We have already studied fullduplex device-to-device communication in [14]. Results there show that most recent full-duplex radios can be considered as suitable for D2D.

In this paper, we investigate full-duplex device-to-device communication in a scenario with multiple antennas in the base station and formulate optimization problems to minimize the sum transmit power of the system. This problem for halfduplex D2D is already studied in [15] and here we consider the full-duplex scenario and compare the result to that of halfduplex, focusing on self-interference cancelation requirements of full-duplex radios.

Rest of the paper is organized as follows. In section II we present the system model. Problem formulation for both uplink and downlink period is discussed in section III. Section IV presents the simulation results. Finally, the conclusion is given in section VI. 


\section{System Model}

We consider a single cell system with one cellular user and one D2D pair. Users are randomly dropped in the cell, considering a maximum distance between D2D users. D2D users are using single antenna full-duplex radios [5] and can switch between half-duplex and full-duplex mode. Cellular user has one antenna and it is sharing radio resources with the D2D pair. Base station is equipped with $M$ antennas. Interference between D2D users and cellular users will be different in uplink and downlink period. In both of the cases, the path loss between D2D user and cellular user is modeled as $G_{0} \cdot(d)^{-\alpha}$ in which $G_{0}$ is the channel gain at a distance of $1 \mathrm{~m}, d$ is the distance between cellular and D2D user and $\alpha$ is the path loss exponent. Also the residual of self-interference is modeled as $\beta \times p_{\mathrm{di}}$, in which $p_{\mathrm{d}}$ is the transmit power of the D2D user $i$ and and $\beta$ depends on the amount of self-interference cancelation. System model for uplink and downlink are presented separately.

\section{A. Uplink Period}

In the uplink period, base station receives interference from D2D transmissions because of radio resource sharing. Also D2D receivers get interference coming from the cellular uplink transmission. Figure 1 shows the system model.

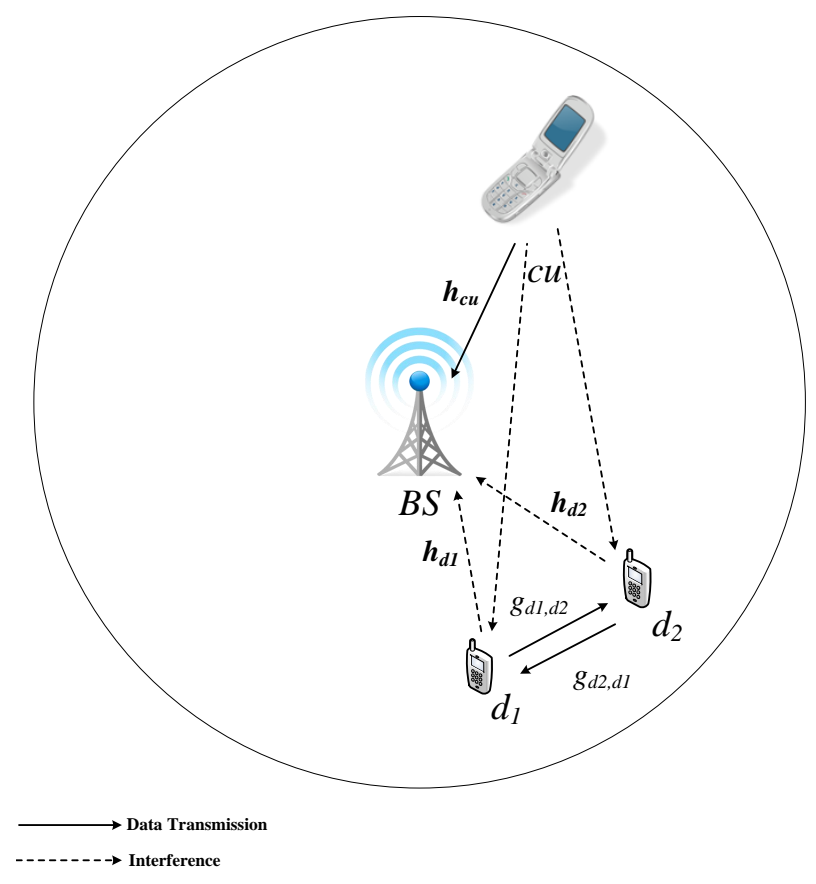

Fig. 1: D2D system model in uplink

Using [15] and equations derived there, we expand the same system equations for full-duplex D2D. The received signal in uplink period in base station is given by

$$
\mathbf{y}_{\mathrm{bs}}=\sqrt{p_{\mathrm{cu}}} \mathbf{h}_{\mathrm{cu}}^{H} d_{\mathrm{cu}}+\sqrt{p_{\mathrm{d} 1}} \mathbf{h}_{\mathrm{d} 1}^{H} d_{\mathrm{d} 1}+\sqrt{p_{\mathrm{d} 2}} \mathbf{h}_{\mathrm{d} 2}^{H} d_{\mathrm{d} 2}+\mathbf{n}_{\mathrm{bs}}
$$

In the above equation, $\mathbf{y}_{\mathrm{bs}}$ is the received signal vector at base station, $p_{\text {cu }}$ is the transmit power of the cellular user, $\mathbf{h}_{\mathrm{cu}} \in \mathbb{C}^{1 \times T}$ is the channel matrix between base station and cellular user and transmit symbol of cellular user is shown by $d_{\mathrm{cu}}$. Transmit powers of D2D users are represented by $p_{\mathrm{d} 1}$ and $p_{\mathrm{d} 2}$ and $\mathrm{D} 2 \mathrm{D}$ transmit symbols are given by $d_{\mathrm{d} 1}$ and $d_{\mathrm{d} 2}$ respectively. Channel gains between D2D users and base station are taken to be $\mathbf{h}_{\mathrm{d} 1}$ and $\mathbf{h}_{\mathrm{d} 2}$. $\mathbf{n}_{\mathrm{bs}}$ is the additive white Gaussian noise vector in base station.

Receive beamforming to estimate cellular user transmit symbol is applied at the base station. Showing the beamforming vector as $\mathbf{w}_{\mathrm{cu}} \in \mathbb{C}^{T}$, the estimated data symbol is

$$
\hat{d}_{\mathrm{cu}}=\mathbf{w}_{\mathrm{cu}}^{H} \mathbf{y}_{\mathrm{bs}}
$$

Considering the interference coming from cellular transmissions and residual of self-interference, received signal at D2D receivers are written as

$$
y_{\mathrm{d} 1}=\sqrt{p_{\mathrm{d} 2}} g_{\mathrm{d} 2, \mathrm{~d} 1} d_{\mathrm{d} 2}+\sqrt{p_{\mathrm{cu}}} g_{\mathrm{cu}, \mathrm{d} 1} d_{\mathrm{cu}}+S I_{1}+n_{\mathrm{d} 1}
$$

$$
y_{\mathrm{d} 2}=\sqrt{p_{\mathrm{d} 1}} g_{\mathrm{d} 1, \mathrm{~d} 2} d_{\mathrm{d} 1}+\sqrt{p_{\mathrm{cu}}} g_{\mathrm{cu}, \mathrm{d} 2} d_{\mathrm{cu}}+S I_{2}+n_{\mathrm{d} 2}
$$

$y_{\mathrm{d} 1}$ and $y_{\mathrm{d} 2}$ are the received signals at D2D users. $g_{\mathrm{d} 1, \mathrm{~d} 2} \in$ $\mathbb{C}$ and $g_{\mathrm{d} 1, \mathrm{~d} 2} \in \mathbb{C}$ are the channel gains between D2D users, $g_{\mathrm{cu}, \mathrm{d} 1}$ and $g_{\mathrm{cu}, \mathrm{d} 2}$ are the gains between D2D receivers and cellular user. $S I_{1}$ and $S I_{2}$ are the residual of the selfinterferences in D2D users 1 and 2 respectively. $n_{\mathrm{d} 1}$ and $n_{\mathrm{d} 2}$ are the additive white Gaussian noise values in D2D receivers. We consider the noise in all the receivers as zero mean Gaussian noise with variance $N_{0}$.

\section{B. Downlink Period}

In downlink period, cellular user receives interference which comes from D2D transmissions. D2D receivers also receive interference from base station. System model is shown in Figure 2.

The received signal $\tilde{y}_{\mathrm{cu}}$ in downlink communication for the cellular user considering the interference due to transmission of D2D users is written as

$$
\tilde{y}_{\mathrm{cu}}=\mathbf{h}_{\mathrm{cu}} \mathbf{m}_{\mathrm{cu}} d_{\mathrm{cu}}+\sqrt{\tilde{p}_{\mathrm{d} 1}} g_{\mathrm{d} 1, \mathrm{cu}} d_{\mathrm{d} 1}+\sqrt{\tilde{p}_{\mathrm{d} 2}} g_{\mathrm{d} 2, \mathrm{cu}} d_{\mathrm{d} 2}+n_{\mathrm{cu}}
$$

In about equation, $\mathbf{m}_{\mathrm{cu}} \in \mathbb{C}^{T}$ is the downlink transmit beamforming vector for the cellular user, $d_{\mathrm{cu}}$ is the data symbol for the cellular user, $\tilde{p}_{\mathrm{d} 1}$ and $\tilde{p}_{\mathrm{d} 2}$ are the powers of the D2D users, $g_{\mathrm{d} 1, \mathrm{cu}}$ and $g_{\mathrm{d} 2, \mathrm{cu}}$ are the channel gains from the D2D transmitters to the cellular user and $n_{\mathrm{cu}}$ is the white Gaussian noise for the cellular user.

The received signals $\tilde{y}_{\mathrm{d} 1}$ and $\tilde{y}_{\mathrm{d} 1}$ at the D2D receivers in the downlink phase are expressed as

$$
\tilde{y}_{\mathrm{d} 1}=\sqrt{\tilde{p}_{\mathrm{d} 2}} g_{\mathrm{d} 2, \mathrm{~d} 1} d_{\mathrm{d} 2}+\mathbf{h}_{\mathrm{d} 1} \mathbf{m}_{\mathrm{cu}} d_{\mathrm{cu}}+S I_{1}+n_{\mathrm{d} 1}
$$

$$
\tilde{y}_{\mathrm{d} 2}=\sqrt{\tilde{p}_{\mathrm{d} 1}} g_{\mathrm{d} 1, \mathrm{~d} 2} d_{\mathrm{d} 1}+\mathbf{h}_{\mathrm{d} 2} \mathbf{m}_{\mathrm{cu}} d_{\mathrm{cu}}+S I_{2}+n_{\mathrm{d} 2}
$$




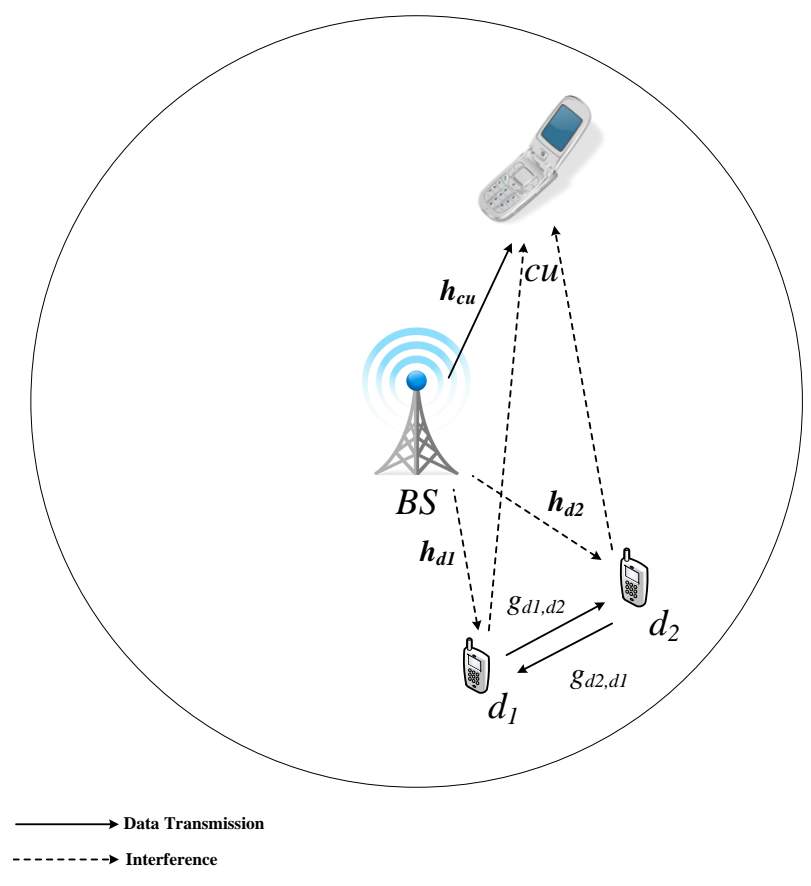

Fig. 2: D2D system model in downlink

Channel vectors form base station to D2D users are shown by $\mathbf{h}_{\mathrm{d} 1}$ and $\mathbf{h}_{\mathrm{d} 2}$. The channel gains between D2D users are represented by $g_{\mathrm{d} 2, \mathrm{~d} 1}$ and $g_{\mathrm{d} 1, \mathrm{~d} 2}$.

\section{PROBLEM Formulation}

In this section we present the optimization problem to minimize the total transmit power of the system. Uplink and downlink period are presented in two sections.

\section{A. Uplink Period}

We formulate the optimization problems to minimize the sum transmit power of the system subject to target signal-tointerference-plus-noise ration (SINR) of each user. Considering uplink period, the optimization problem is formulated as

$$
\begin{array}{ll}
\underset{p_{\mathrm{cu}}, p_{\mathrm{d} 1}, p_{\mathrm{d} 2}, \mathbf{w}_{\mathrm{cu}}}{\min } & p_{\mathrm{cu}}+p_{\mathrm{d} 1}+p_{\mathrm{d} 2} \\
\text { s. t. } & \frac{p_{\mathrm{cu}}\left|\mathbf{w}_{\mathrm{cu}}^{H} \mathbf{h}_{\mathrm{cu}}^{H}\right|^{2}}{p_{\mathrm{d} 1}\left|\mathbf{w}_{\mathrm{cu}}^{H} \mathbf{h}_{\mathrm{d} 1}^{H}\right|^{2}+p_{\mathrm{d} 2}\left|\mathbf{w}_{\mathrm{cu}}^{H} \mathbf{h}_{\mathrm{d} 2}^{H}\right|^{2}+N_{0}} \geq \gamma_{\mathrm{cu}} \\
& \frac{p_{\mathrm{d} 1}\left|g_{\mathrm{d} 1, \mathrm{~d} 2}\right|^{2}}{p_{\mathrm{cu}}\left|g_{\mathrm{cu}, \mathrm{d} 2}\right|^{2}+S I_{2}+N_{0}} \geq \gamma_{\mathrm{d} 2} \\
& \frac{p_{\mathrm{d} 2}\left|g_{\mathrm{d} 2, \mathrm{~d} 1}\right|^{2}}{p_{\mathrm{cu}}\left|g_{\mathrm{cu}, \mathrm{d} 1}\right|^{2}+S I_{1}+N_{0}} \geq \gamma_{\mathrm{d} 1}
\end{array}
$$

In this equation, $\gamma_{\mathrm{cu}}, \gamma_{\mathrm{d} 2}$ and $\gamma_{\mathrm{d} 2}$ are target SINR for cellular and D2D users respectively.

We propose a joint uplink power control and receive beamforming algorithm to solve (8). This algorithm is presented for solving the uplink problem for conventional cellular communications, however, in this work we extend this algorithm to D2D system. To solve this optimization problem, we set the Lagrangian of (8) to zero (w.r.t. $\mathbf{w}_{\mathrm{cu}}$ ) and then after applying some re-arrangement optimal power control and receive beamforming can be calculated using the following fixed-point iterations. Detailed explanation of how to solve the problem and obtain the equations are explained in [16]. For cellular users transmit power is obtained from the following formula

$$
p_{\mathrm{cu}}[t+1]=\frac{\gamma_{\mathrm{cu}}}{\mathbf{h}_{\mathrm{cu}}\left(N_{0} \mathbf{I}+p_{\mathrm{d} 1}[t] \mathbf{h}_{\mathrm{d} 1}^{H} \mathbf{h}_{\mathrm{d} 1}+p_{\mathrm{d} 2}[t] \mathbf{h}_{\mathrm{d} 2}^{H} \mathbf{h}_{\mathrm{d} 2}\right)^{-1} \mathbf{h}_{\mathrm{cu}}^{H}}
$$

For D2D users the transmit power is calculated using

$$
\begin{aligned}
& p_{\mathrm{d} 1}[t+1]=\frac{\gamma_{\mathrm{d} 2}}{g_{\mathrm{d} 1, \mathrm{~d} 2}\left(N_{0}+p_{\mathrm{cu}}[t] g_{\mathrm{cu}, \mathrm{d} 2}^{H} g_{\mathrm{cu}, \mathrm{d} 2}+S I_{1}\right)^{-1} g_{\mathrm{d} 1, \mathrm{~d} 2}^{H}}(10) \\
& p_{\mathrm{d} 2}[t+1]=\frac{\gamma_{\mathrm{d} 1}}{g_{\mathrm{d} 2, \mathrm{~d} 1}\left(N_{0}+p_{\mathrm{cu}}[t] g_{\mathrm{cu}, \mathrm{d} 1}^{H} g_{\mathrm{cu}, \mathrm{d} 1}+S I_{2}\right)^{-1} g_{\mathrm{d} 2, \mathrm{~d} 1}^{H}}
\end{aligned}
$$

While solving this problem we consider the residual of selfinterference to be equal to the noise in the receivers. Linear MMSE beamforming in the base station can be performed to design the receive beamformer:

$$
\mathbf{w}_{\mathrm{cu}}=\frac{\hat{\mathbf{w}}_{\mathrm{cu}}}{\left\|\hat{\mathbf{w}}_{\mathrm{cu}}\right\|_{2}}
$$

$$
\hat{\mathbf{w}}_{\mathrm{cu}}=\left(N_{0} \mathbf{I}+p_{\mathrm{d} 1} \mathbf{h}_{\mathrm{d} 1}^{H} \mathbf{h}_{\mathrm{d} 1}+p_{\mathrm{d} 2} \mathbf{h}_{\mathrm{d} 2}^{H} \mathbf{h}_{\mathrm{d} 2}\right)^{-1} \mathbf{h}_{\mathrm{cu}}^{H}
$$

The proposed uplink power control and receive beamforming approach is summarized in Algorithm 1.

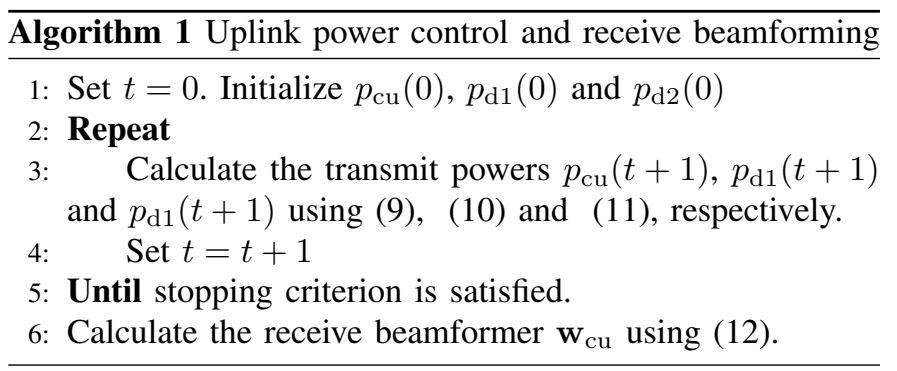

The Algorithm 1 can be used in centralized approach. The centralized approach works such that the BS has CSI of all users. In addition, the BS receives inter-user channel information from D2D transmitters. The BS uses this information to find the optimal transmit power of cellular user and D2D transmitter.

\section{B. Downlink Communication}

In this section we formulate and solve the optimization problem in downlink. The problem is to minimize the total transmit power of the system subject to SINR constraints in users. Following equations show the optimization problem. 
TABLE I: Simulation Parameters

$$
\begin{aligned}
& \min _{\tilde{p}_{\mathrm{d} 1}, \tilde{p}_{\mathrm{d} 2}, \mathbf{m}_{\mathrm{cu}}}\left\|\mathbf{m}_{\mathrm{cu}}\right\|_{2}^{2}+\tilde{p}_{\mathrm{d} 1}+\tilde{p}_{\mathrm{d} 2} \\
& \text { s. t. } \quad \frac{\left|\mathbf{h}_{\mathrm{cu}} \mathbf{m}_{\mathrm{cu}}\right|^{2}}{\tilde{p}_{\mathrm{d} 1}\left|g_{\mathrm{d} 1, \mathrm{cu}}\right|^{2}+\tilde{p}_{\mathrm{d} 2}\left|g_{\mathrm{d} 2, \mathrm{cu}}\right|^{2}+N_{0}} \geq \gamma_{\mathrm{cu}} \\
& \frac{\tilde{p}_{\mathrm{d} 1}\left|g_{\mathrm{d} 1, \mathrm{~d} 2}\right|^{2}}{\left|\mathbf{h}_{\mathrm{d} 2} \mathbf{m}_{\mathrm{cu}}\right|^{2}+S I_{2}+N_{0}} \geq \gamma_{\mathrm{d} 2} \\
& \frac{\tilde{p}_{\mathrm{d} 2}\left|g_{\mathrm{d} 2, \mathrm{~d} 1}\right|^{2}}{\left|\mathbf{h}_{\mathrm{d} 1} \mathbf{m}_{\mathrm{cu}}\right|^{2}+S I_{1}+N_{0}} \geq \gamma_{\mathrm{d} 1}
\end{aligned}
$$

This problem can be formulated as an SOCP problem. Considering $\hat{p}_{\mathrm{d} 1}=\sqrt{\tilde{p}_{\mathrm{d} 1}}$ and $\hat{p}_{\mathrm{d} 2}=\sqrt{\tilde{p}_{\mathrm{d} 2}}$ now the optimization problem is written as [17]

$$
\begin{array}{ll}
\operatorname{min.} & q \\
\text { s. t. } & \left\|\begin{array}{c}
\hat{p}_{\mathrm{d} 1} g_{\mathrm{d} 1, \mathrm{cu}} \\
\hat{p}_{\mathrm{d} 2} g_{\mathrm{d} 2, \mathrm{cu}} \\
\sqrt{N_{0}}
\end{array}\right\|_{2} \leq \sqrt{\frac{1}{\gamma_{\mathrm{cu}}}} \mathbf{h}_{\mathrm{cu}} \mathbf{m}_{\mathrm{cu}} \\
& \left\|\begin{array}{c}
\mathbf{h}_{\mathrm{d} 1} \mathbf{m}_{\mathrm{cu}} \\
S I_{1} \\
\sqrt{N_{0}}
\end{array}\right\|_{2} \leq \sqrt{\frac{1}{\gamma_{\mathrm{d} 1}} \hat{p}_{\mathrm{d} 2} g_{\mathrm{d} 2, \mathrm{~d} 1}} \\
& \left\|\begin{array}{c}
\mathbf{h}_{\mathrm{d} 2} \mathbf{m}_{\mathrm{cu}} \\
S I_{2} \\
\sqrt{N_{0}}
\end{array}\right\|_{2} \leq \sqrt{\frac{1}{\gamma_{\mathrm{d} 1}}} \hat{p}_{\mathrm{d} 1} g_{\mathrm{d} 1, \mathrm{~d} 2} \\
& \left\|\mathbf{m}_{\mathrm{cu}}\right\|_{\hat{p}_{\mathrm{d} 1}} \leq q \\
\hat{p}_{\mathrm{d} 2}
\end{array} \|_{2}
$$

If channel state information (CSI) is available at transmitter, this problem can be solved using CVX. Transmit power of D2D users and precoder in base station are found with this problem.

\section{Simulation Results}

In this section we present numerical simulation results to study the effect of residual of self-interference in the performance of the system. We consider an OFDMA based system, in which each user has two LTE resource blocks (360 khz). In the simulations we calculate the rate of the D2D link using Shannon's capacity formula for both half-duplex and full-duplex mode. In half-duplex mode we consider that only $d_{2}$ is transmitting and $d_{1}$ is receiving the signal. Also there is no residual of self-interference in half-duplex mode. Presenting the rate of D2D users in half-duplex and full-duplex mode by $R_{H D}$ and $R_{F D}$, the rates are given by

$$
\begin{gathered}
R_{H D}=\log _{2}\left(1+S I N R_{H D}\right) \\
R_{F D}=\log _{2}\left(1+S I N R_{d 1}\right)+\log _{2}\left(1+S I N R_{d 2}\right)
\end{gathered}
$$

While calculating the optimal transmit power and beamformer, we consider the residual of self-interference to be equal to background noise for simplicity. Then in the simulation results we plot the rate ratio of full-duplex over halfduplex mode, $R_{F D} / R_{H D}$ to study the effect of the residual

\begin{tabular}{c|c}
\hline \hline Parameter & Value \\
\hline \hline Number of Antennas in BS (M) & 4 \\
\hline Cell Radios & $500 \mathrm{~m}$ \\
\hline Maximum D2D Distance & $25 \mathrm{~m}$ \\
\hline$\alpha$ & 4 \\
\hline Noise Figure at BS & $2 \mathrm{~dB}$ \\
\hline Noise Figure at CU & $9 \mathrm{~dB}$ \\
\hline D2D Path Loss Model & $148+40 \log (\mathrm{d}[\mathrm{km}])$ \\
\hline BS to CU Path Loss Model & $128.1+36.7 \log (\mathrm{d}[\mathrm{km}])$ \\
\hline Noise spectral density & $-174 \mathrm{dBm} / \mathrm{Hz}$ \\
\hline
\end{tabular}

of self-interference on the performance of the D2D link. Table I shows the simulation parameters.

We run the simulations for different amounts of selfinterference cancelation in full-duplex D2D radios. In uplink period for 5 different SINR targets, the rate ratio is plotted versus the amount of self-interference cancelation in Figure 3. This figure shows that for SINR target such as $0 \mathrm{~dB}$ or $5 \mathrm{~dB}$, full-duplex radios with $100 \mathrm{~dB}$ self-interference cancelation provide double the throughput compared to that of halfduplex radios. Increasing the amount of SINR target causes an increase in D2D transmit powers and thererfore canceling the self-interference to the noise-floor becomes harder and more self-interference cancelation is required.

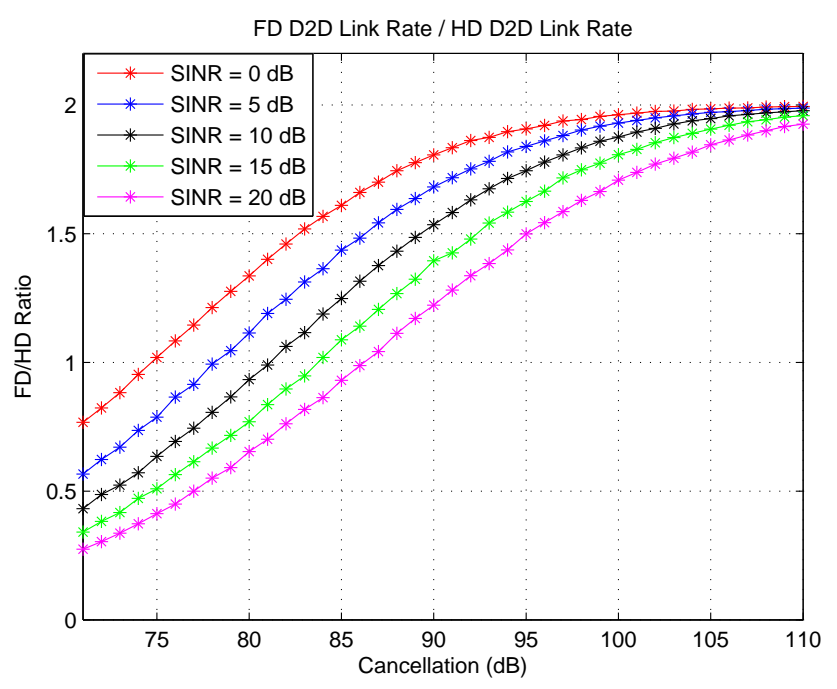

Fig. 3: FD/HD rate ratio in uplink period for different SINR targets

For uplink period, in Figure 4 we show the rate ratio for different amounts of self-interference cancelation based on the distance of D2D users. This figure shows that the smaller the distance between D2D users, the better the full-duplex radios perform. This is due to small transmit power of D2D users. As seen from the figure, for $70 \mathrm{~dB}$ self-interference cancelation, full-duplex radios have very bad performance because residual of self-interference is too large compared the noise and D2D interferences. However, with $110 \mathrm{~dB}$ selfinterference cancelation, for all the distances up to 25 meters, full-duplex radios are able to double the throughput of the D2D link. 


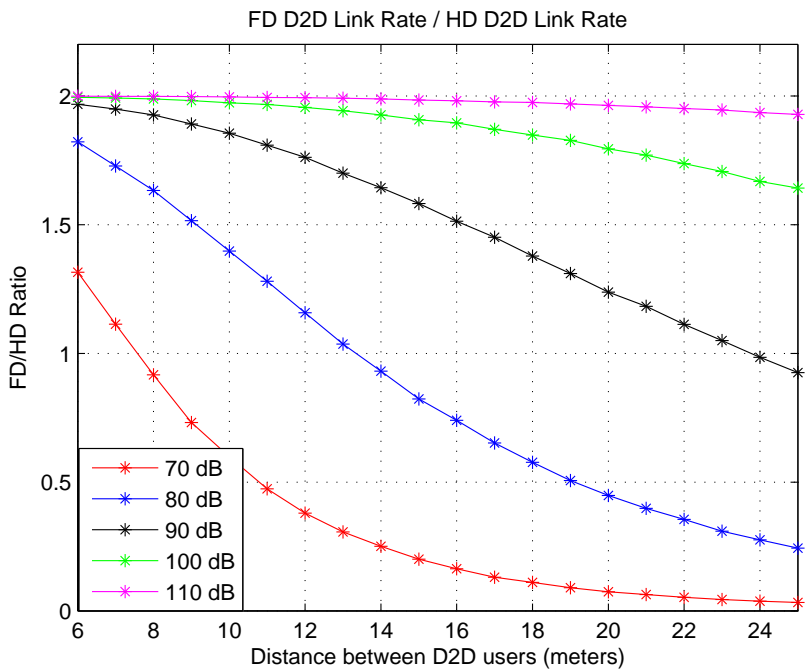

Fig. 4: FD/HD rate ratio in uplink based on distance of D2D users for SINR target $10 \mathrm{~dB}$

In downlink communications, we perform the same simulations as done in uplink. Fig. 5 shows the rate ratio based on the amount of self-interference cancelation for different SINR targets. It is seen that in the downlink period, for our system model, full-dulex performance is worse than the uplink period. Full-duplex radios need more self-interference cancelation to outperform the half-duplex radios. The reason behind is that in uplink, there was no method to limit the interference coming from cellular users to D2D users, and that interference increases the noise-plus-interference level both in half-duplex and full-duplex radios. For higher noise-plusinterference level, full-duplex radios with less self-interference cancelation are able to double the throughput compared to half-duplex. In the downlink period, since base station is performing beamforming, the amount of interference on D2D is smaller and noise-plus-interference level is smaller.

Also one point that is seen Figure 5 is that for $0 \mathrm{~dB}$ SINR target and $110 \mathrm{~dB}$ self-interference cancelation, FD provides more than the double the half-duplex rate. This originates from the fact that when two users are transmitting, they have different interference levels coming from cellular transmissions. When the half-duplex user has high interference, there is a possibility that one of the full-duplex users has smaller interference and the rate of full-duplex mode can be more than double the half-duplex.

Figure 6 shows the rate ratio based on distance of D2D users in downlink period. Simulations are done for different amounts of self-interference cancelation. It is shown that for $110 \mathrm{~dB}$ self-interference cancelation, almost for all the distances fullduplex doubles the throughput compared to half-duplex.

From all the simulation results shown above it is seen that full-duplex radios with $110 \mathrm{~dB}$ self-interference cancelation are able to provide double the throughput compared to halfduplex radios.

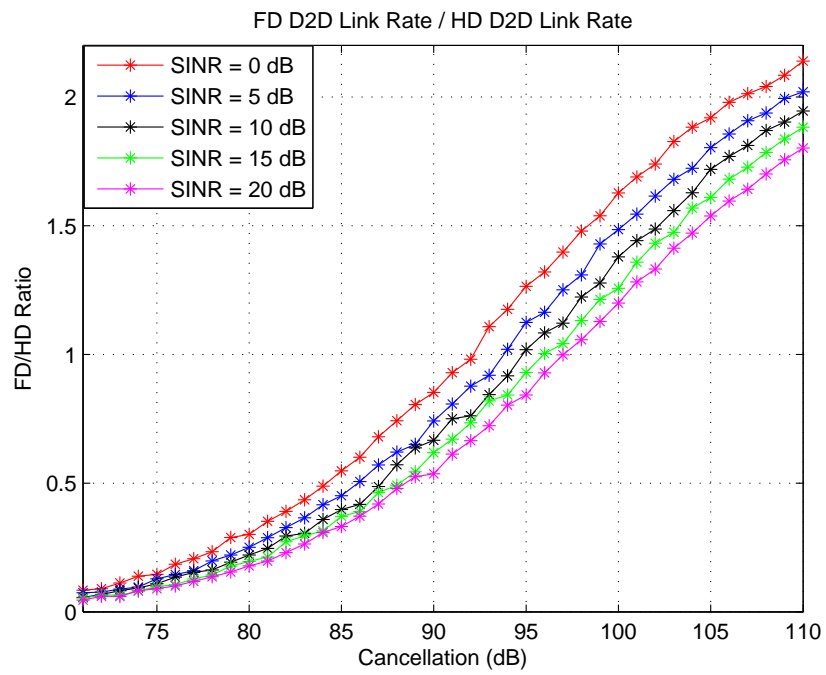

Fig. 5: FD/HD rate ratio in downlink period for different SINR targets

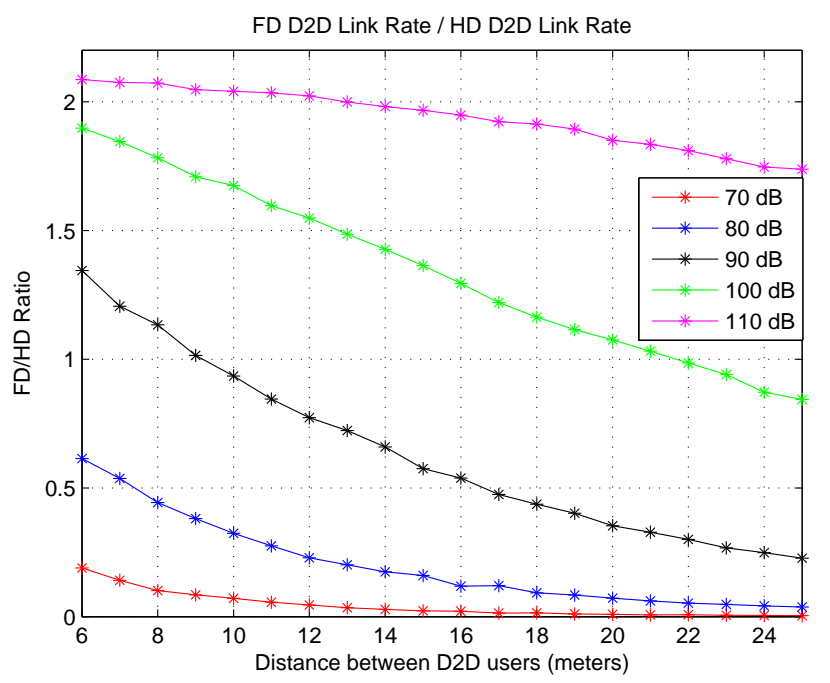

Fig. 6: FD/HD rate ratio in downlink based on distance of D2D users for SINR target $10 \mathrm{~dB}$

\section{CONCLUSIONS}

In this paper, full-duplex radios are considered in devce-todevice communication in cellular systems. We have studied the performance of the system for different amounts of selfinterference cancelation, for full duplex radios and compared the results to half-duplex radios. The system model consists of a pair of underlay D2D users that are utilizing single antenna full-duplex radios, one single antenna cellular user and a base station with multiple antennas. Optimization problem is the sum-power minimization of the system subject to SINR target. In uplink period, fixed point iterations are used to find the optimal transmit powers of the users. In downlink, problem is formed as SOCP problem and solved optimally using CVX in Matlab. Full-duplex over half-duplex rate ratio is illustrated in simulations results for different amounts of self-interference 
cancelation. Also results based on distance between D2D are shown. Different SINR targets also effect the results of the simulation. Simulation results show that full-duplex radios can be implemented in $\mathrm{D} 2 \mathrm{D}$ and most recent radios can provide double the throughput.

\section{REFERENCES}

[1] J. I. Choi, M. Jain, K. Srinivasan, P. Levis, and S. Katti, "Achieving single channel, full duplex wireless communication," in Proceedings of the Sixteenth Annual International Conference on Mobile Computing and Networking, ser. MobiCom '10. New York, NY, USA: ACM, 2010, pp. 1-12.

[2] E. Aryafar, M. A. Khojastepour, K. Sundaresan, S. Rangarajan, and M. Chiang, "Midu: Enabling mimo full duplex," in Proceedings of the 18th Annual International Conference on Mobile Computing and Networking, ser. Mobicom '12. New York, NY, USA: ACM, 2012, pp. 257-268.

[3] M. Duarte, C. Dick, and A. Sabharwal, "Experiment-driven characterization of full-duplex wireless systems," Wireless Communications, IEEE Transactions on, vol. 11, no. 12, pp. 4296-4307, 2012.

[4] M. Jain, J. I. Choi, T. Kim, D. Bharadia, S. Seth, K. Srinivasan, P. Levis, S. Katti, and P. Sinha, "Practical, real-time, full duplex wireless," in Proceedings of the 17th Annual International Conference on Mobile Computing and Networking, ser. MobiCom '11. New York, NY, USA: ACM, 2011, pp. 301-312. [Online]. Available: http://doi.acm.org/10.1145/2030613.2030647

[5] D. Bharadia, E. McMilin, and S. Katti, "Full duplex radios," SIGCOMM Comput. Commun. Rev., vol. 43, no. 4, pp. 375-386, Aug. 2013.

[6] M. Duarte, A. Sabharwal, V. Aggarwal, R. Jana, K. Ramakrishnan, C. Rice, and N. Shankaranarayanan, "Design and characterization of a full-duplex multiantenna system for wifi networks," Vehicular Technology, IEEE Transactions on, vol. 63, no. 3, pp. 1160-1177, March 2014.

[7] D. Bharadia and S. Katti, "Full duplex mimo radios," in Proceedings of the 11th USENIX Symposium on Networked Systems Design and Implementation (NSDI 14). Seattle, WA: USENIX, 2014, pp. 359-372. [Online]. Available: http://blogs.usenix.org/conference/nsdi14/ technical-sessions/bharadia

[8] K. Doppler, M. Rinne, C. Wijting, C. Ribeiro, and K. Hugl, "Deviceto-device communication as an underlay to lte-advanced networks," Communications Magazine, IEEE, vol. 47, no. 12, pp. 42-49, Dec 2009.

[9] P. Jänis, C.-H. Yu, K. Doppler, C. B. Ribeiro, C. Wijting, K. Hugl, O. Tirkkonen, and V. Koivunen, "Device-to-device communication underlaying cellular communications systems." 2009.

[10] K. Doppler, M. Rinne, P. Janis, C. Ribeiro, and K. Hugl, "Deviceto-device communications; functional prospects for lte-advanced networks," in Communications Workshops, 2009. ICC Workshops 2009. IEEE International Conference on, June 2009, pp. 1-6.

[11] K. Doppler, C.-H. Yu, C. Ribeiro, and P. Janis, "Mode selection for device-to-device communication underlaying an lte-advanced network," in Wireless Communications and Networking Conference (WCNC), 2010 IEEE, April 2010, pp. 1-6.

[12] S. Hakola, T. Chen, J. Lehtomaki, and T. Koskela, "Device-to-device communication in cellular network - performance analysis of optimum and practical communication mode selection," in Wireless Communications and Networking Conference (WCNC), 2010 IEEE, April 2010, pp. $1-6$.

[13] H. Min, J. Lee, S. Park, and D. Hong, "Capacity enhancement using an interference limited area for device-to-device uplink underlaying cellular networks," Wireless Communications, IEEE Transactions on, vol. 10, no. 12 , pp. 3995-4000, 2011.

[14] S. Ali, N. Rajatheva, and M. Latva-aho, "Full duplex device-to-device communication in cellular networks," in Networks and Communications (EuCNC), 2014 European Conference on, June 2014, pp. 1-5.

[15] A. Ghazanfari, A. Tolli, and H. Pennanen, "Sum power minimization for cellular systems with underlay d2d communications," in Cognitive Radio Oriented Wireless Networks and Communications (CROWNCOM), 2014 9th International Conference on, June 2014, pp. 45-50.

[16] H. Dahrouj and W. Yu, "Coordinated beamforming for the multicell multi-antenna wireless system," IEEE Trans. Wireless Commun, vol. 9, no. 5, pp. 1748-1759, May. 2010.

[17] A. Wiesel, Y. C. Eldar, and S. Shamai, "Linear precoding via conic optimization for fixed MIMO receivers," IEEE Trans. Signal Process., vol. 54, no. 1, pp. 161-176, Jan. 2006. 